\title{
EL CORAZÓN DE LOS PUEBLOS DE COLONIZACIÓN
}

\author{
Antonio Álvaro Tordesillas y Alberto Meiss
}

The heart of the Villages of Colonization

Boletín Académico. Revista de investigación y arquitectura contemporánea

Escuela Técnica Superior de Arquitectura. Universidade da Coruña

elSSN 2173-6723

unw.boletinacademico.com

Número 3 (2013)

Páginas 37-48

Fecha de recepción 31.10.2012

Fecha de aceptación 22.01.2013

https://doi.org/10.17979/bac.2013.3.0.995

\section{Resumen}

Los centros de los pueblos levantados por el INC en los ańos cincuenta y sesenta en España, son uno de los mejores casos donde estudiar la evolución que la arquitectura y el urbanismo de nuestro país estaban experimentando, libres ya del imposible problema sobre el "estilo español". Fueron el banco de pruebas donde ensayar criterios de diseño más libres y orgánicos, trazados espacialmente más flexibles y articulados entre sí, consecuentes con la historia y el lugar, dimensionados según la medida humana y potenciando la relación recíproca entre sus habitantes... Lugares que, con el transcurso de los años, dibujaron la modernidad.

\section{Abstract}

The village centers raised by the INC in the fifties and sixties in Spain, are one of the best cases in which study the evolution that the architecture and the city planning of our country were experiencing, freed from the impossible problem of the "Spanish style". They were the testing bench in which were tested free and organic designs, more flexible and articulated sketches, consistent with the history and place, sized according to the human measure and enhancing the reciprocal report between its inhabitants... Places that, over the years, drew the modernity.

\section{Palabras clave}

Colonización, Centro cívico, Urbanismo, Análisis gráfico, Core

\section{Keywords}

Colonization, Civic center, Town planning, Graphical analysis, Core 


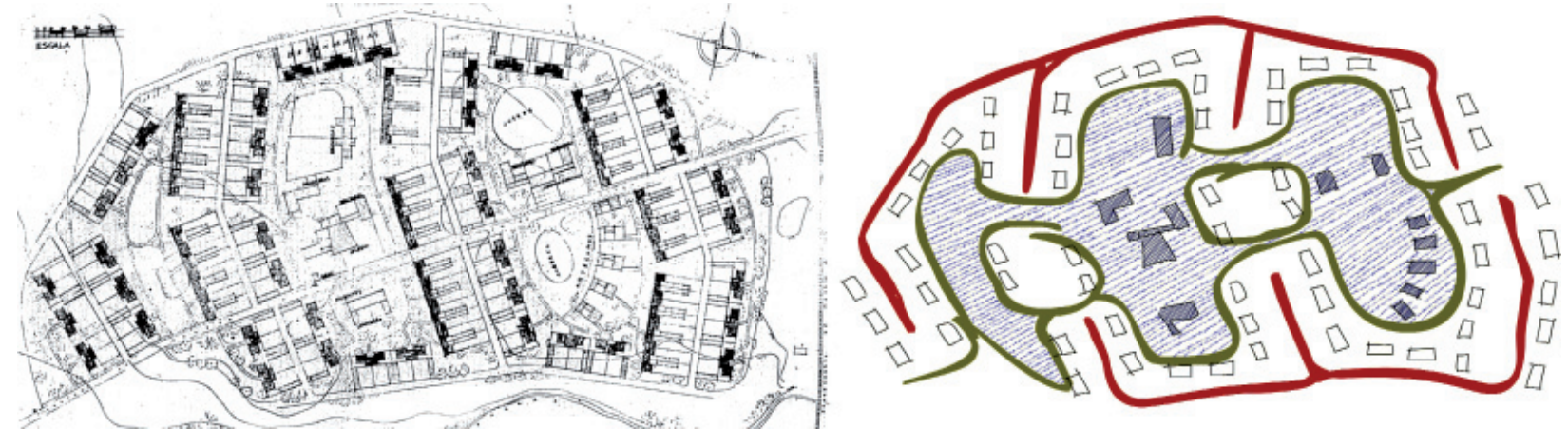

1 José Luis Fernández del Amo Moreno, Torres de Salinas (Toledo), 1951

\section{LA IDEA DE CORAZÓN}

Los años cincuenta suponen para las poblaciones levantadas por el Instituto Nacional de Colonización una moderna y revolucionaria transformación formal. Sus circulaciones, sus relaciones interiores o la disposición de sus manzanas son algunas de las causas. Modelos importados norteamericanos, como la manzana $R a-$ dburn, ingleses como las primeras New Towns, israelíes como los kibbutzim, son referentes incuestionables'. Pero son sus centros cívicos, sin duda alguna, las piezas que fundamentalmente sufren $-\mathrm{y}$ a su vez causandicha transformación. Centros que hasta entonces se concebían próximos al referente clásico de Plaza Mayor y que verían transformar su forma y esencia de un modo radical. Centros que convenimos denominar corazones, al aproximarlos a la idea de core definida en el VIII CIAM, celebrado en Hoddesdon (Reino Unido) en 1951, y aplicada al primero de los cinco grados que en él se establecieron: esto es, el poblado rural' ${ }^{2}$. Entonces se debatió la necesidad de crear lugares centrales de los que participasen frecuentemente sus habitantes, dando forma y expresión a sus sentimientos:

«El core es un lugar donde la gente se puede reunir para recrearse y relacionarse, ya sea en el espacio abierto de una pequeña comunidad o en el más grande centro de la Ciudad. El core es un artefacto: un elemento esencial del planeamiento urbano elaborado por el hombre. Es la expresión de la mente colectiva y el espíritu de la comunidad y da significado y forma a la ciudad misma $»^{3}$.
Por muchos considerado el último, este CIAM suponía una reacción a la conflictiva Carta de Atenas que propugnaba, como sabemos, desde un estandarte de partir de cero, una ciudad nueva cuyo modelo era claramente opuesto al histórico.

Un año más tarde, en España se redactaba el Manifiesto de la Alhambra, cuyos objetivos versaban sobre intenciones similares: el ataque al monumentalismo mediante la búsqueda de un módulo humano, y la atención al entorno circundante de la manera más respetuosa.

¿Fue este ambiente de reflexión el que recogió el INC a la hora de indicar las directrices de ordenación de los pueblos de colonización? Al plantearse los centros cívicos teóricos, ¿se pensaba según la actual corriente humanista del octavo CIAM, del Manifiesto de la Alhambra, o aún se hacía al modo de la antigua Dirección General de Regiones Devastadas?

Hemos de procurar no confundir automáticamente los centros cívicos de nuestros pueblos con sus corazones. Es importante descubrir si los primeros son realmente espacios de libre relación e intercambio sociocultural entre sus vecinos o meras escenografías donde son manipulados como títeres bajo una falsa libertad. Es común aceptar toda esta parafernalia en las poblaciones realizadas por Regiones Devastadas; sin embargo, ¿es esto trasladable a Colonización?

El INC surge de manera más autónoma que la DGRD, ajeno a la polémica sobre el estilo español. Autonomía que le permite convertirse en un banco de 


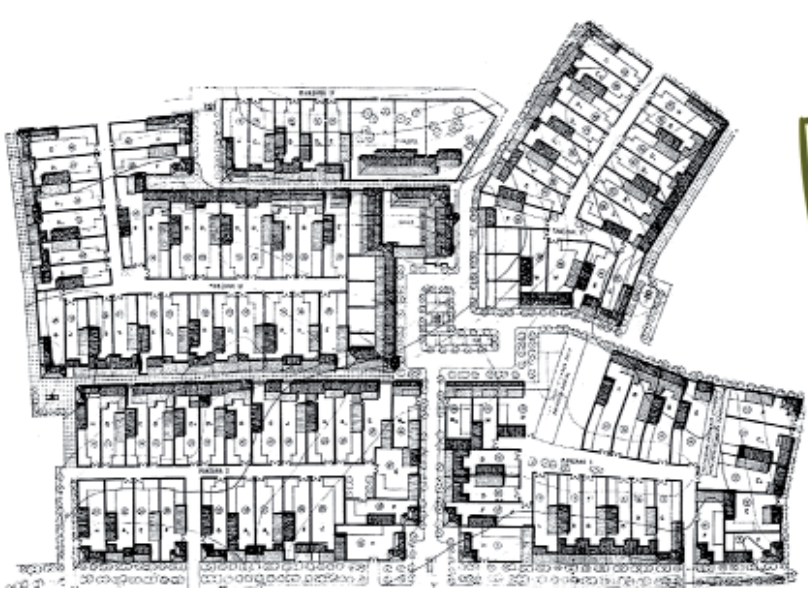

2 José Tamés Alarcón, Torre de la Reina (Sevilla), 1952

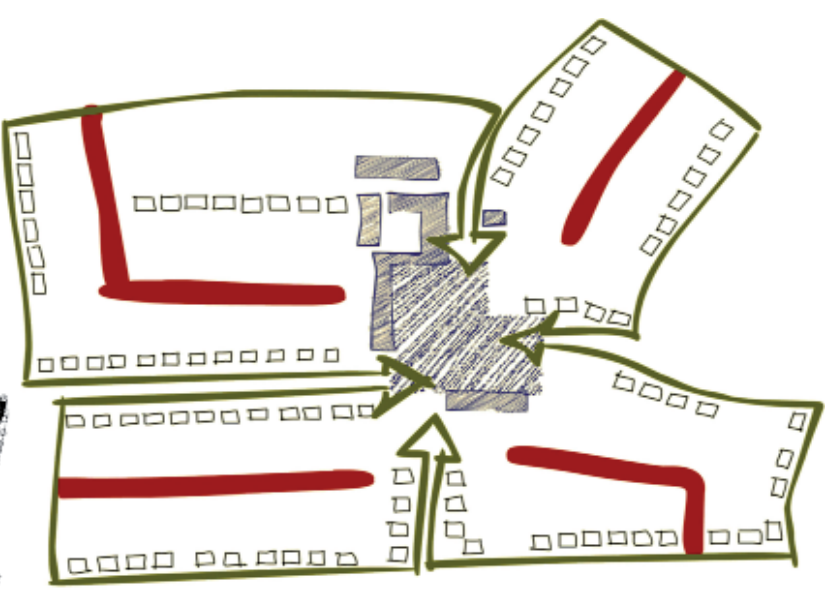

pruebas — según afirma Alejandro de la Sota- para que una generación de jóvenes profesionales, educados en plena desorientación académica, puedan ejercitar aquello de que "para acertar, debemos precisamente olvidar todo, casi todo lo poco que sabemos $»^{4}$.

Veremos que se puede responder en ambos sentidos. Por un lado, todos los centros cívicos de los pueblos de colonización son lugares de reunión y escenarios de manifestación de sus moradores. Por otro, son referentes determinantes de sus trazados urbanos; pero aquí, sin embargo, encontraremos que tan sólo algunos son capaces de convertir orgánicamente la relación centroplaza, definidora de un trazado tradicional, en corazón-pueblo, definidora de un trazado moderno. Serán estos casos los que trataremos: aquéllos en los que el acercamiento al core tenga mayor sentido que el mero uso analógico del término.

\section{PUEBLOS CON CORAZÓN}

Resulta significativo comprobar cómo, tanto el congreso de Hoddesdon como el encuentro de Granada, se celebraron inaugurando la década de los cincuenta, pues fue ésta especialmente fructífera para el INC, no sólo ya en el número de sus realizaciones sino también en la transformación cualitativa de éstas. No en vano ésta se conoce como la década de oro de la colonización española. Sin embargo, afirmar que el cambio de década supone un punto de inflexión es, quizá, forzar una intencionada metáfora que no aporta más que el hecho de relacionar este cambio con el de mentalidad que se venía manifestando en la arquitectura y el urbanismo internacionales.
En el año 1951, José Luis Fernández del Amo, arquitecto funcionario del INC desde 1947, proponía al Servicio de Arquitectura del INC el novedoso y revolucionario trazado de Torres de Salinas, en la provincia de Toledo. Un proyecto donde, además de contemplarse la separación de circulaciones entre personas y animales de un modo claro y sincero, se propone un trazado orgánico, respetuoso con la naturaleza existente y libre de rigideces formales (Fig. 01). Del Amo se adelantaba a su tiempo, y dicho Servicio adoptó una actitud lógicamente reaccionaria. Aquello rompía todos los esquemas, y además incomodaba al propio jefe del Servicio, José Tamés Alarcón, que por aquel entonces se encontraba ultimando el proyecto de Torre de la Reina, en Sevilla; un pueblo que pretendía reunir la inercia de trabajo y resultados que hasta entonces se habían logrado en el Instituto, constituyéndose casi como modelo. Modelo que todavía sería una referencia para el diseño de muchos otros posteriores.

Y aunque el trazado de Torre de la Reina introducía interesantes novedades -especialmente con la particular interpretación de la manzana Radburn y sus calles de carros - conceptualmente, distaba mucho del de Fernández del Amo (Fig. 02). «Tamés defendía un tipo de pueblo mezcla de racionalidad circulatoria y reproducción pintoresca ${ }^{5}$. La Memoria del proyecto era una perfecta exposición didáctica de esa teoría: por un lado, y en consonancia con los principios sittescos, se planteaban calles quebradas, perspectivas rematadas por algún edificio singular o manzanas compuestas con una cierta variedad de tipos arquitectónicos; por otro, aparecía un esquema de circulaciones separadas 

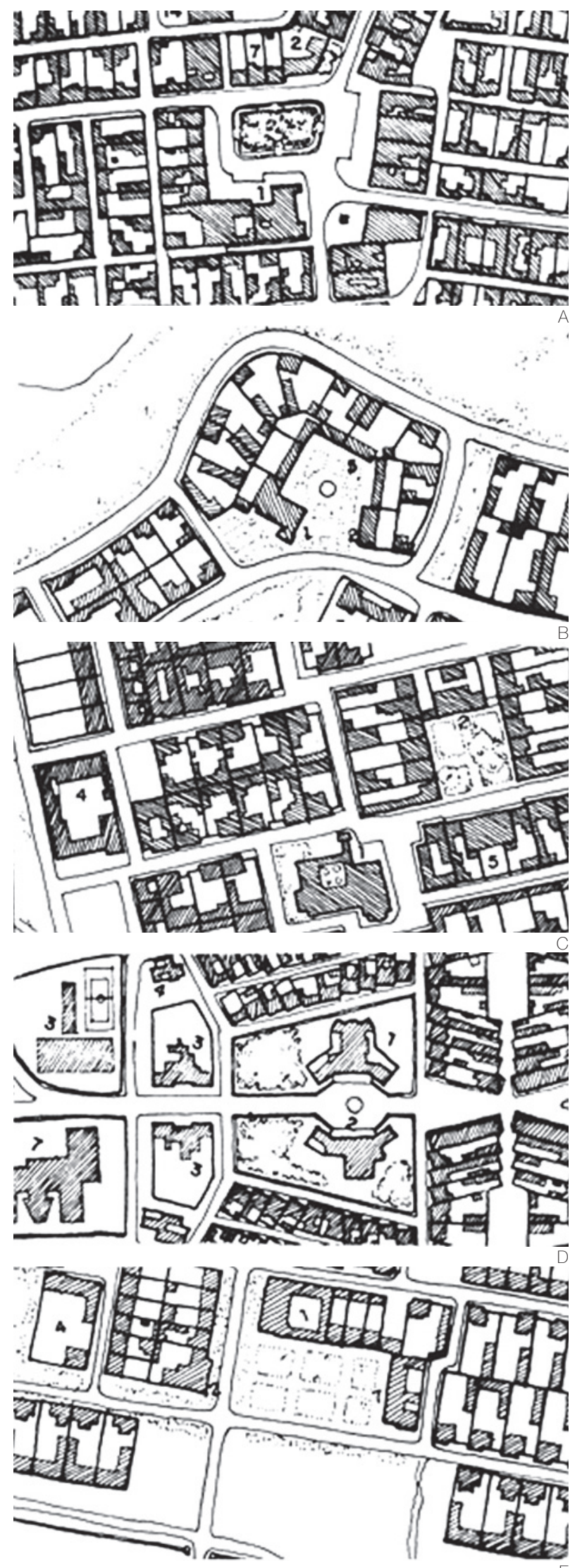

3 Cinco tipos de plaza: A. La situada en el centro del pueblo; B. El esquema de plaza cerrada; C. La articulación de pequeñas plazas; D. La plaza que abarca a sus edificios singulares exentos en su interior; y E. La plaza abierta y una arquitectura de gusto popular, pero simplificada en sus detalles y racionalizada en las plantas.

Habitualmente se ha emparejado - y simplificado-el análisis del centro cívico de los pueblos con el estudio de sus plazas mayores. Generalmente la evolución de éstas repercute en la de aquéllos, pero no es exclusiva.

Villanueva y Leal, en el estudio que desde la UIAI realizaron para el Ministerio de Agricultura hace ya más de dos décadas ${ }^{6}$, hacían precisamente esto, describiendo cinco tipos de plaza: la situada en el centro del pueblo, la plaza cerrada, la articulación de pequeñas plazas, la plaza que abarca a sus edificios singulares quedando exentos en su interior, y la plaza abierta (Fig. 03). Esta reducción impide un acercamiento más próximo a la idea de core y dificulta una visión más generosa de dichos lugares: la que incluye su relación con la totalidad del pueblo, su influencia en el trazado urbano, etc.

Con el transcurso de los años, las plazas se transforman en corazones más orgánicos y modernos en su ordenación y forma. Fijémonos en las fechas de distintos pueblos: El Torno (1943), Gimenells (1943) (Fig. 04), Láchar (1944) y Valdelacalzada (1947) responden al tipo de plaza central, donde se reconoce claramente el esquema tradicional de plaza y calle mayor, más afín al de Tamés pero, sobre todo, al de la Dirección General de Regiones Devastadas. Regiones Devastadas, un organismo próximo al INC pero con ideas y objetivos diferentes, de planteamientos mucho más rígidos y tradicionales, valoraba de manera especial la plaza mayor como configuradora única del centro cívico (Fig. 05)7 .

Colonización supo ir desprendiéndose de esa epidérmica aproximación a lo tradicional en sus edificaciones, al partir de planteamientos más libres, funcionales y experimentales. Centro cívico y trama urbana se relacionan orgánicamente generando espacios de aproximación, apertura, articulación y referencia. Los edificios públicos serán en menor cantidad e importancia, pues el carácter y sentido son totalmente diferentes de los de aquéllos de Regiones Devastadas: protagonistas de ordenar una colonización interior, no estandartes de un nuevo régimen.

Aún conviviendo con el modelo de Tamés, surgen nuevos planteamientos: Esquivel (1952), Gévora (1954), Villalba de Calatrava (1955), Entrerríos (1955), Llanos del Sotillo (1956), Vegaviana (1959), Lácara (1961), Cañada del Agra (1962), La Vereda (1963), Miraelrío (1964), El Priorato (1964), Maribáńez (1964), Setefilla (1965) o Sacramento (1965). 


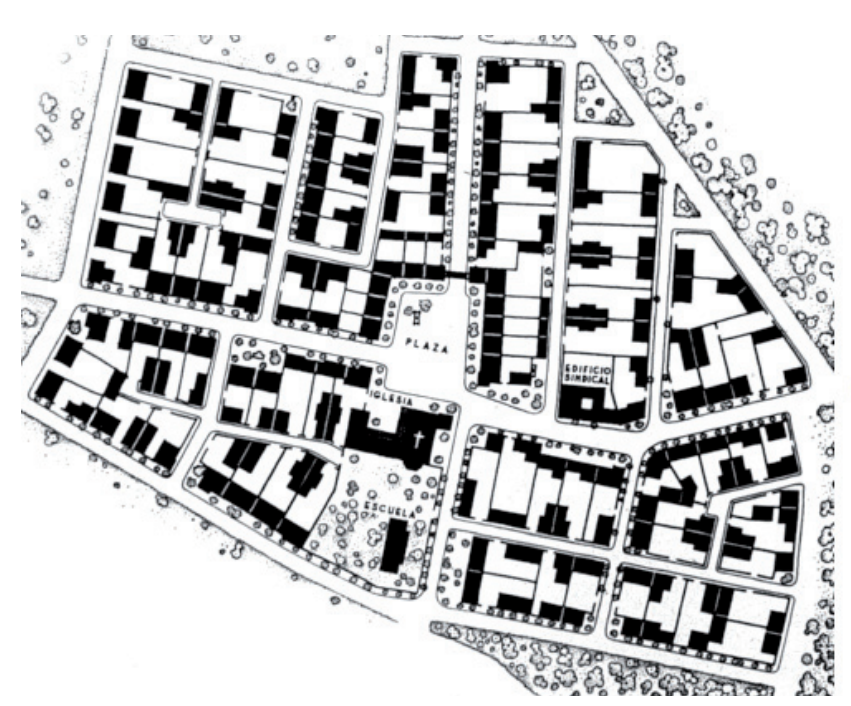

4 Alejandro de la Sota Martínez, Gimenells (Lleida), 1943

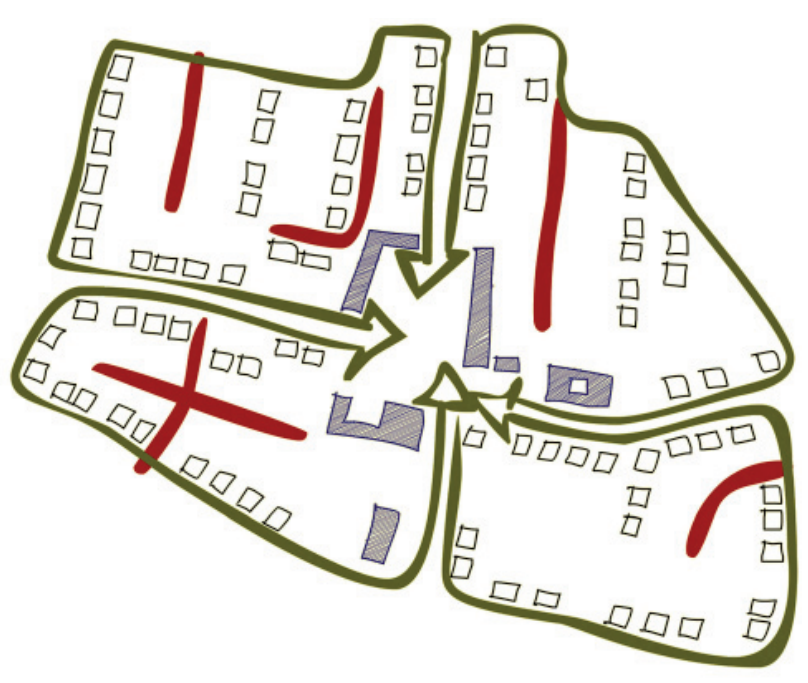

Este último es una supermanzana con una cuidada organización y separación de circulaciones (Fig. 06). Las calles peatonales que desembocan en las plazoletas se disponen en forma de turbina, compartiendo una de ellas, que las articula, y donde se encuentran los edificios singulares: iglesia, escuelas, edificio administración y cooperativas. En Sacramento (Sevilla) nos encontramos ante un formidable ejemplo de cómo la tradicional plaza mayor debilita su carácter oficial posibilitando así una mayor difusión cotidiana del espacio público. La organicidad de esta original plaza hace del centro cívico de Sacramento un corazón expresado mediante la interrelación de las edificaciones y los espacios que generan. El encadenamiento de espacios cerrados que definen su centro, de calles y plazas, la diferenciación de circulaciones, etc., del que hacen gala este pueblo lo convierten en un hito; y por su fecha, además, en el colofón del Instituto.

¿A qué tipología, de las cinco enunciadas, responde a la plaza de Sacramento? A ninguna. Y, repito, no sólo porque no se asemeje formalmente a ninguna de ellas, sino por lo complejo de la relación que mantiene con su entorno inmediato y con la ordenación general del pueblo.

Un caso singular es Llanos del Sotillo (Jaén) (Fig. 07). Un pueblo donde se realiza una profunda revisión del alojamiento rural que afecta a la vivienda, la calle y al espacio público. Su planta es todo un diagrama de funcionamiento. Llanos del Sotillo se organiza sobre una malla ortogonal muy rígida en planta y alzado. La mayor parte del programa de vivienda se proyectó en planta alta, dejando libre la planta baja y creando unos puentes sobre las calles peatonales. La separación de circulaciones es patente. Y su centro cívico adopta el mismo criterio, generando una insólita plaza mayor techada. Mediante unas rampas se accede a la planta que la cubre, donde se encuentran los edificios públicos: cantina, cooperativa, escuelas, viviendas de maestros e iglesia. En Llanos del Sotillo, la configuración del lugar central se traslada a las calles del pueblo, de manera que éstas participan del carácter de aquél. Aquí se reproduce esa relación entre las partes y el todo que buscamos.

En pueblos como Gévora, Maribáñez, Entrerríos o Vegaviana, los edificios públicos aparecen exentos dentro del espacio mayor que supone el centro cívico (Fig. 08). A pesar de que el recinto desaparezca como cerrado, o delimitado, sus edificios mantienen su singularidad, y los referentes simbólicos, como la torre de la iglesia, continuarán trabajando de la misma manera. El que se trate, casi siempre, de espacios peatonales, contribuye al mejor entendimiento del espacio y, por tanto, a su buen uso. Mas no se trata sólo de unos edificios exentos dispuestos en un espacio amplio, sino que todo el pueblo se convierte en contenedor, en escenografía para alojarlos. Es la identificación plaza-pueblo.

Lácara (Badajoz) o Cañada del Agra (Albacete) (Fig. 09) significan un paso más allá en el desmembramiento formal del espacio tradicional de la plaza mayor. El corazón, además de cumplir con todo lo dicho hasta el momento, se abre hacia el campo, incorporando a la trama urbana las parcelas de cultivo. No sólo se pro- 


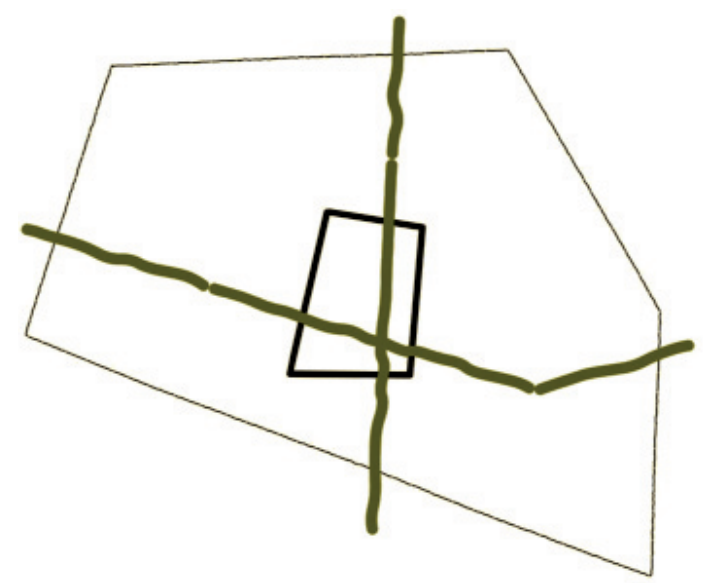
la plaza mayor y la calle principa

duce la relación entre los vecinos del pueblo, sino de éstos con sus propios campos de cultivo. Urbe y campo se comunican, otorgando un carácter más complejo al core del pueblo.

El caso más rotundo, en este sentido, es el de Esquivel (Sevilla) (Fig. 10). No en vano, según señala su autor, Alejandro de la Sota, se trata de un experimento, lo que explica su precocidad. Sin lugar a dudas el espacio resultante de desarrollar la plaza es uno de los inventos de Sota en este proyecto. Plaza que, al desarrollarse, define una fachada exterior y, a su vez, arroja la iglesia y el ayuntamiento, solos, al espacio más sobresaliente del pueblo. Desde luego, es de suponer que un espacio tan decididamente moderno, en una fecha todavía tan temprana, habría de verse censurado, de nuevo, por el Instituto. De ahí que los árboles que hoy pueblan el espacio de esta plaza no fueron sino un mero acuerdo entre éste y el arquitecto.

La plaza abierta, en general, tampoco abandona su función de centro cohesionador, y en la mayoría de las ocasiones se mantiene en el mismo centro geométrico del pueblo, dejando libre todo el abanico visual que desde él se abarca. Desde el corazón del pueblo, sus moradores pueden sentarse a charlar observando $-\mathrm{y}$ vigilando- sus propias parcelas de labor.

Y aún nos encontramos un caso diferente. Pueblos como La Vereda o Miraelrío, más parecen grandes cortijos; o incluso uno de esos kibbutzim israelíes (Fig. 11) $)^{8}$. La referencia a estos asentamiento judíos no se hace sólo por su similitud formal (compárese con Nahalal), sino

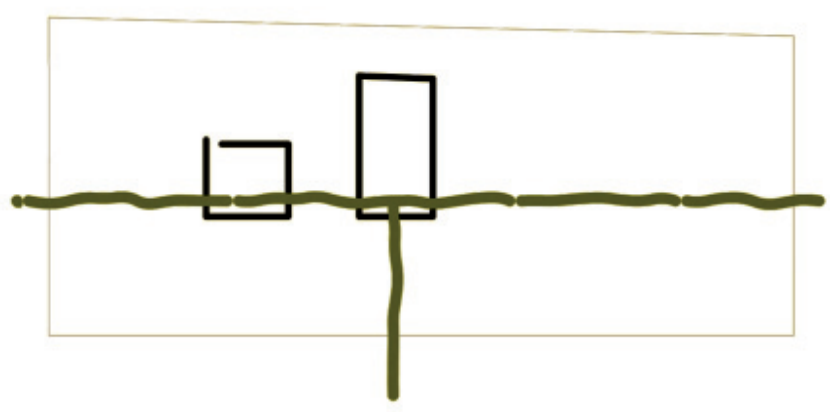

por su concepción del centro cívico. En el centro de la población, en un prado, se sitúa el edificio central que hace las veces de comedor, sala de juntas, lectura, eventos sociales, etc. El prado sirve a la totalidad de la población como lugar de esparcimiento. En verano, lo usan como lugar para proyectar cine al aire libre.

En La Vereda (Sevilla), el trazado del poblado se define mediante dos grandes patios, uno circunscrito por las viviendas de los colonos y el otro, porticado, rodeado por edificios públicos. Ambos se articulan a través del edificio de la administración, «evitando así el sistema urbanístico de plazas y calles»?. Rupturas deliberadas en el lienzo que forman las fachadas de las viviendas permiten a los vecinos prolongar sus vistas hacia los campos de cultivo. Miraelrío (Jaén) se organiza mediante un anillo de viviendas que encierran una espina dorsal de equipamientos abiertos mediante patios al mediodía. Miraelrío es un gran ejemplo de reflexión sobre temas como la escala del espacio abierto, de lo público y de lo privado. ¿Y la plaza? O bien el pueblo se ha apropiado de la ella, o bien ésta de aquél.

\section{LOS EDIFICIOS PÚBLICOS}

Hay dos edificios en los centros de los pueblos de especial relevancia arquitectónica. Se trata de las iglesias y de las escuelas. Ambos equipamientos cumplían una función simbólica importante, además de la propia. Por ello el INC les otorga programas y tamaños generosos. Sus torres, elemento de referencia tanto como hito visual desde la lejanía como adalid moral a seguir y cultivar, adquieren un papel especial. 

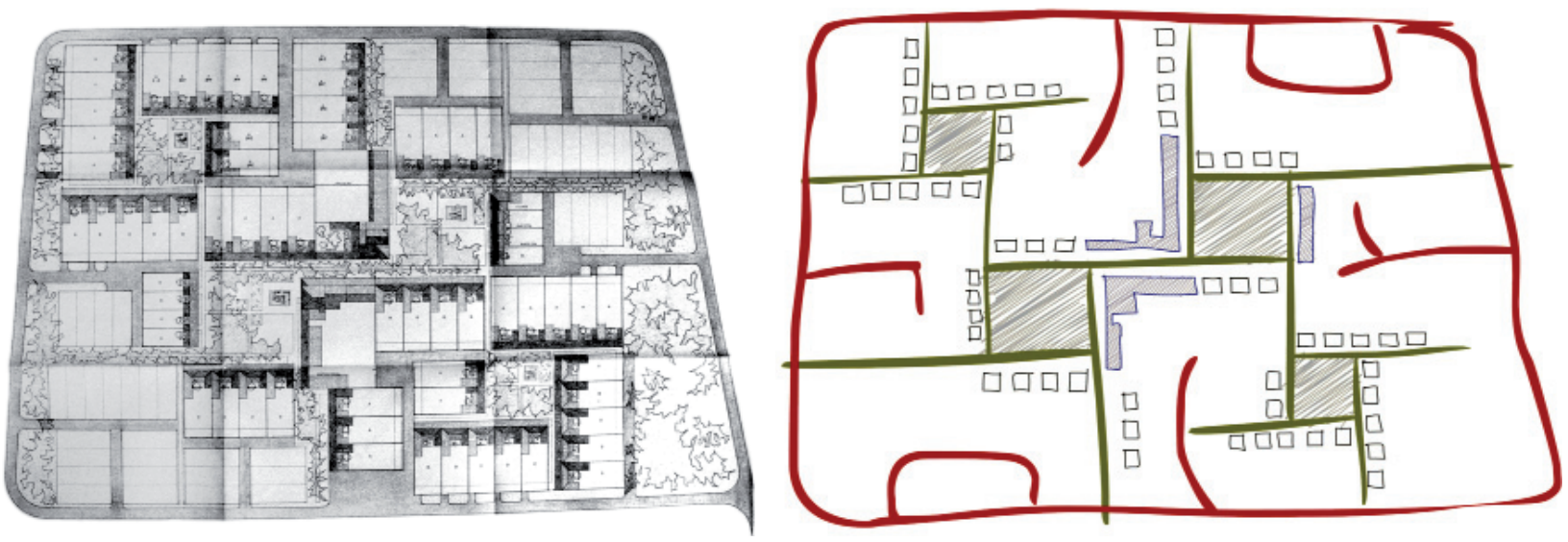

6 Fernando de Terán Troyano, Sacramento (Sevilla), 1965
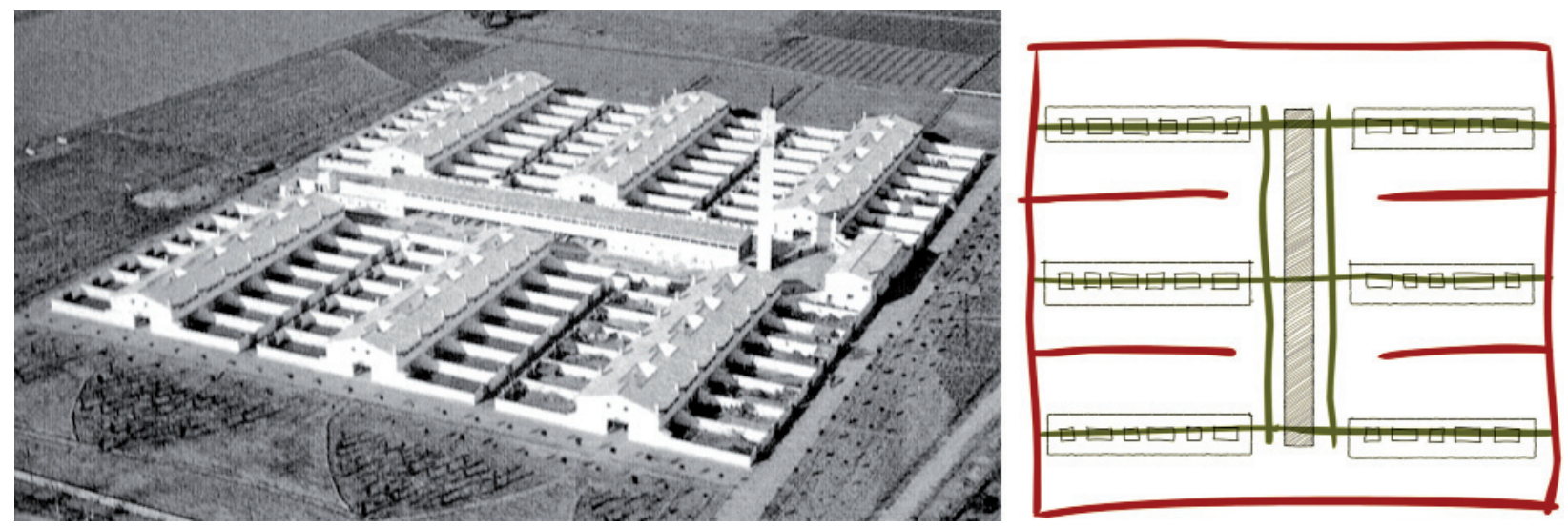

7 José Antonio Corrales Gutiérrez, Llanos del Sotillo (Jaén), 1956
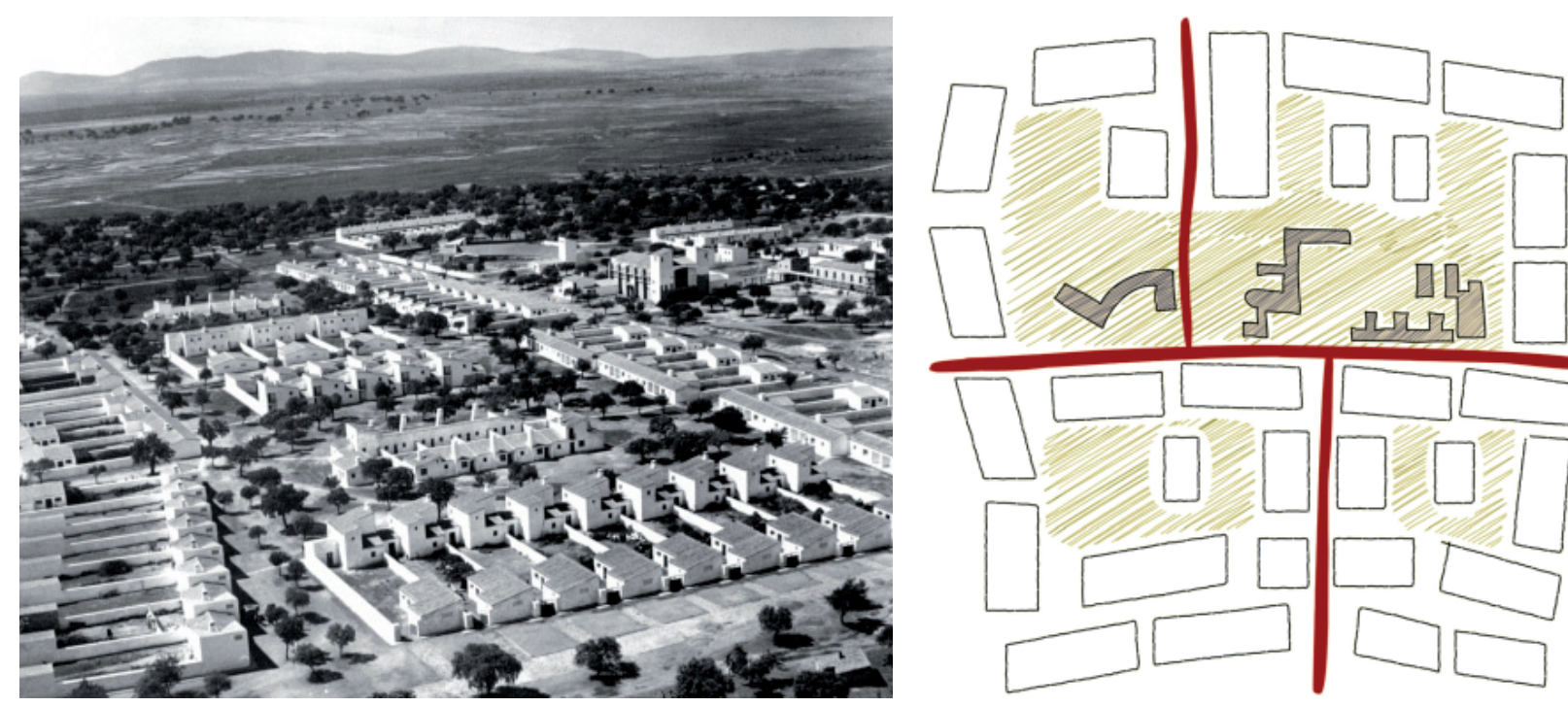

8 José Luis Fernández del Amo Moreno, Vegaviana (Cáceres), 1959

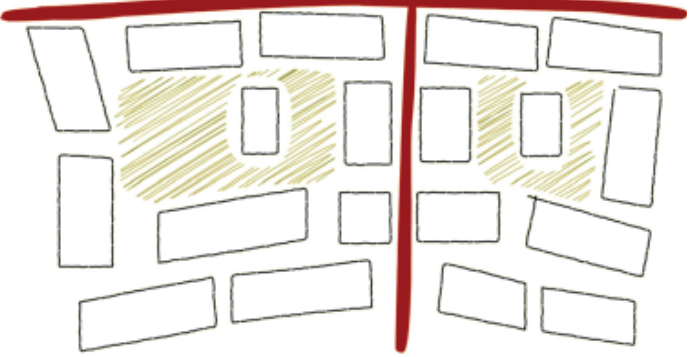



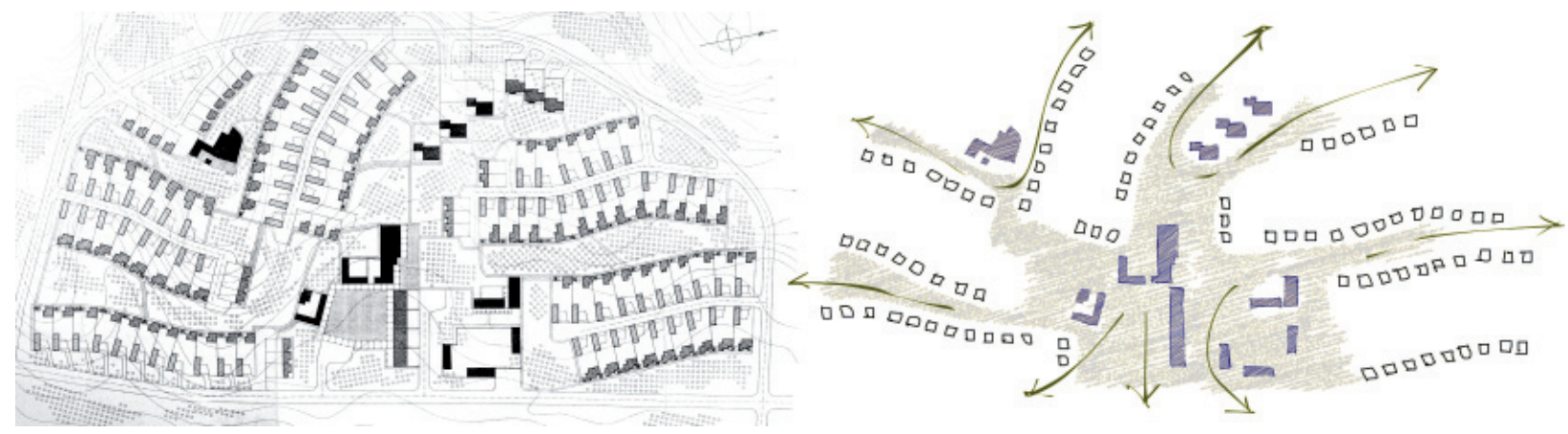

En la España de posguerra, la relación más importante que tuvo la arquitectura con el mundo sacro pasó por el concurso para la terminación de la catedral de la Almudena en Madrid, la construcción de la capilla del Espíritu Santo de Miguel Fisac, o el concurso que ganaron Francisco Javier Sáenz de Oíza y Luis Laorga para el santuario de Aranzazu en Guipúzcoa. «Aranzazu significó (...) el inicio de la modernización del arte sacro ${ }^{10}$. Modernización de la que echó mano el que, en febrero de 1952, sería director del Museo de Arte Contemporáneo, José Luis Fernández del Amo, al incorporar en el diseño de las iglesias de Colonización artistas de otras disciplinas. Basta con acercarse a algunos pueblos. Por ejemplo, en Villalba de Calatrava (Ciudad Real) la fachada de la iglesia se convierte en retablo donde Manuel Hernández Mompó transforma el cerramiento de la nave en un gran mural. En el interior de la nave aparecen, además, un vía crucis y un retablo abstractos de Pablo Serrano, en talla de madera y elementos de forja de hierro. Podríamos pensar también en el fresco del altar de Esquivel, o en el mural cerámico de Antonio Rodríguez Valdivieso y el vía crucis en mosaico de gres de Antonio González Suárez, en Vegaviana (Cáceres) (Fig. 12).

Sin embargo, junto a estos ejemplos de abstracción y modernidad, también hubo otros que se afrontaron desde una visión más elemental y figurativa, como el fresco del altar de Villafranco del Delta (en la actualidad Poblenou del Delta, Tarragona), de José Borobio Ojeda, o las vidrieras de Rafael Arévalo, en Torre de la Reina, las de José Luis Sánchez Fernández en Fuentes Nuevas (León), etc.
Con todo, la incorporación de otras artes en la escena arquitectónica fue especialmente característica en el Instituto y objeto de su propia modernidad. Y además, es de remarcar la reveladora transformación iconográfica que estos artistas realizaron diez años antes del Concilio Vaticano II.

Por otro lado, la renovación espacial también es importante. Las primeras iglesias del INC responden a características formales locales y tradicionales: El Torno, Gimenells o El Temple, por citar algunas. Se trata de templos no muy diferentes de las iglesias de los viejos pueblos castellanos, con una nave longitudinal que producía una silueta clara y una fachada plana: baptisterio a los pies, y presbiterio y altar centrados en el ábside.

El Concilio de 1962 estimuló una evolución arquitectónica más apresurada. Las plantas dejaban su tipo basilical para transformarse en geometrías más atrevidas:plantas circulares, cuadradas, con el presbiterio situado en diversas posiciones... Los volúmenes también gozan de esta nueva manumisión, dejándose ver principalmente en las cubiertas y lucernarios: Nava de Campana, Entrerríos, Marismillas o Setefilla (Fig. 13).

La importancia de las escuelas en la colonización se refleja en su número. Superan las setecientas (para algo menos de trescientos pueblos), y ello es debido a que este equipamiento determinaba fuertemente la permanencia en el poblado. Además, el mejor modo de luchar contra el analfabetismo era precisamente evitando que se produjera; así, la asistencia a clase era obligatoria. 

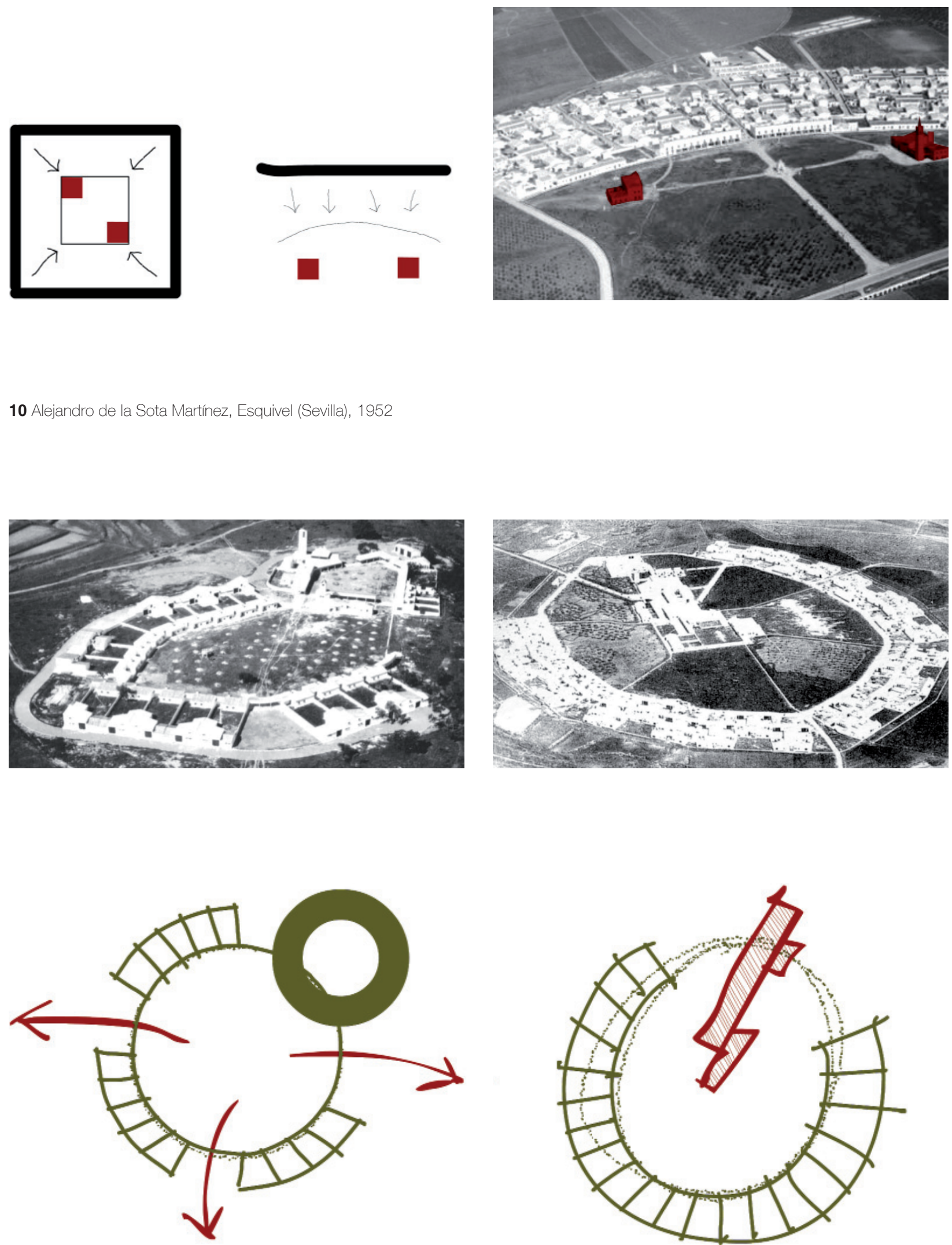

11 José Luis Fernández del Amo Moreno, La Vereda (Sevilla), 1963 y Miraelrío (Jaén), 1964 

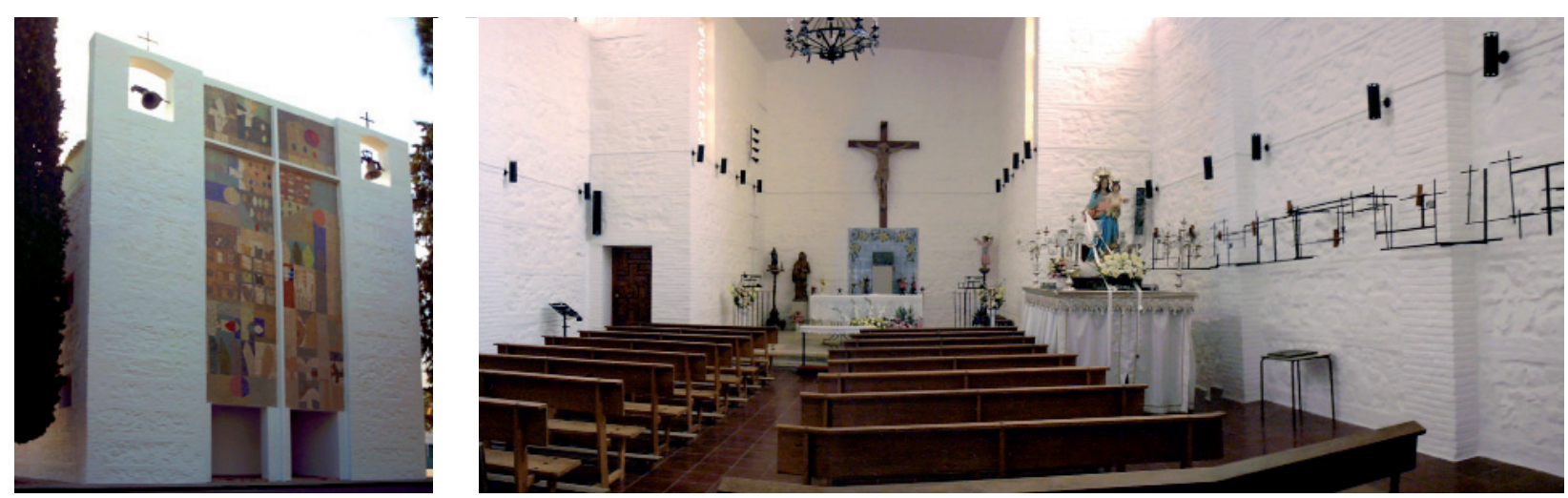

Apuntamos brevemente cómo, en los pueblos más pequeños, las escuelas compartían su espacio con otros usos, principalmente el de capilla. Estas pequeñas aulas eran mixtas, salvo en los pueblos de mayor entidad, donde se duplicaban diferenciando a los alumnos por sexos, incluso en el patio de recreo.

La evolución de estas piezas pasa por la transformación en el modo de entender el edificio en su conjunto. Se trata de pequeñas edificaciones exentas que traslucen su volumen sin artificios ni postizos añadidos. De nuevo las cubiertas, la sección, los huecos de ventanas, etc., nos muestran un proceso de cambio que, poco a poco, va abandonando aquella imagen regionalista por otra más moderna. El edificio pesado y masivo se convierte ahora en liviano, como vemos, por ejemplo, en Villalba de Calatrava. Se llega, incluso, a la industrialización de módulos válidos para poblaciones de climas muy diferentes, como sucede en Nuevos Francos (Salamanca) y en El Trobal (Sevilla) (Fig. 14).

\section{CONCLUSIÓN}

En el análisis y definición del centro cívico de los Pueblos de Colonización nos hemos acercado a la idea de core en cuanto a los poblados rurales se refiere; es decir, como mínima unidad social satisfactoria. Dicha idea sugiere un centro-corazón que ha de ser referente para su población, tanto como lugar donde los pobladores desarrollen sus actividades de relación, como en la definición espacial entre aquél y la totalidad del poblado. Si bien todos los centros cívicos de los pueblos de colonización son capaces de lo primero, no lo son de lo segundo. Aquellos que hemos visto en este artículo llevan la idea hasta sus últimas consecuencias, determinando, incluso, tanto el trazado urbano como las maneras de usarlo y aprovecharlo: separación de circulaciones, ruptura de perspectivas, fachadas de manzana, organicidad de trazados, relación campohabitantes, etc.

Estos pueblos pudieron proyectarse y construirse gracias a la libertad de maniobras de que disfrutó el Instituto Nacioanl de Colonización. En este organismo se ensayaron criterios de diseño y dimensionado para unas necesidades reales, donde la medida humana fuera la que rigiera todos sus elementos, y donde se potenciara la relación recíproca entre los vecinos. Y algunos pueblos, quizá los mejores, quizá los más modernos, quizá aquellos de autores más denodados y destacados, consiguieron ese carácter de referente, de centro nodal y, a la vez, dibujaron una flexibilidad espacial, una articulación con el todo que los hizo especialmente modernos.

En el transcurso de los años en los que el Instituto fue colonizando el campo, se produjo, en la evolución de la arquitectura en España, el cambio necesario y suficiente que permitió pensar y proyectar corazones para sus pueblos y ciudades. Y el INC aportó parte del testimonio construido de esa evolución, de esa modernización. 

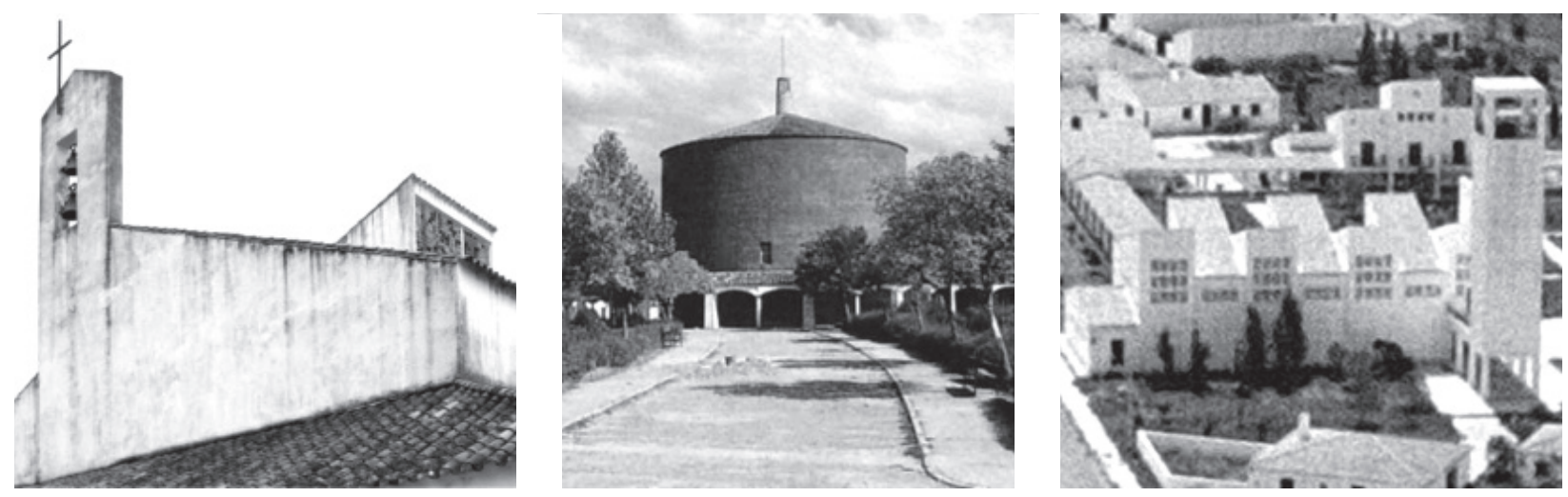

13 De izquierda a derecha: Fernando de Terán Troyano, Setefilla (Sevilla), 1965; Alejandro de la Sota Martínez, Entrerríos (Badajoz), 1956; Jesús Hernández Martínez-Arcos, Marismillas (Sevilla), 1965)
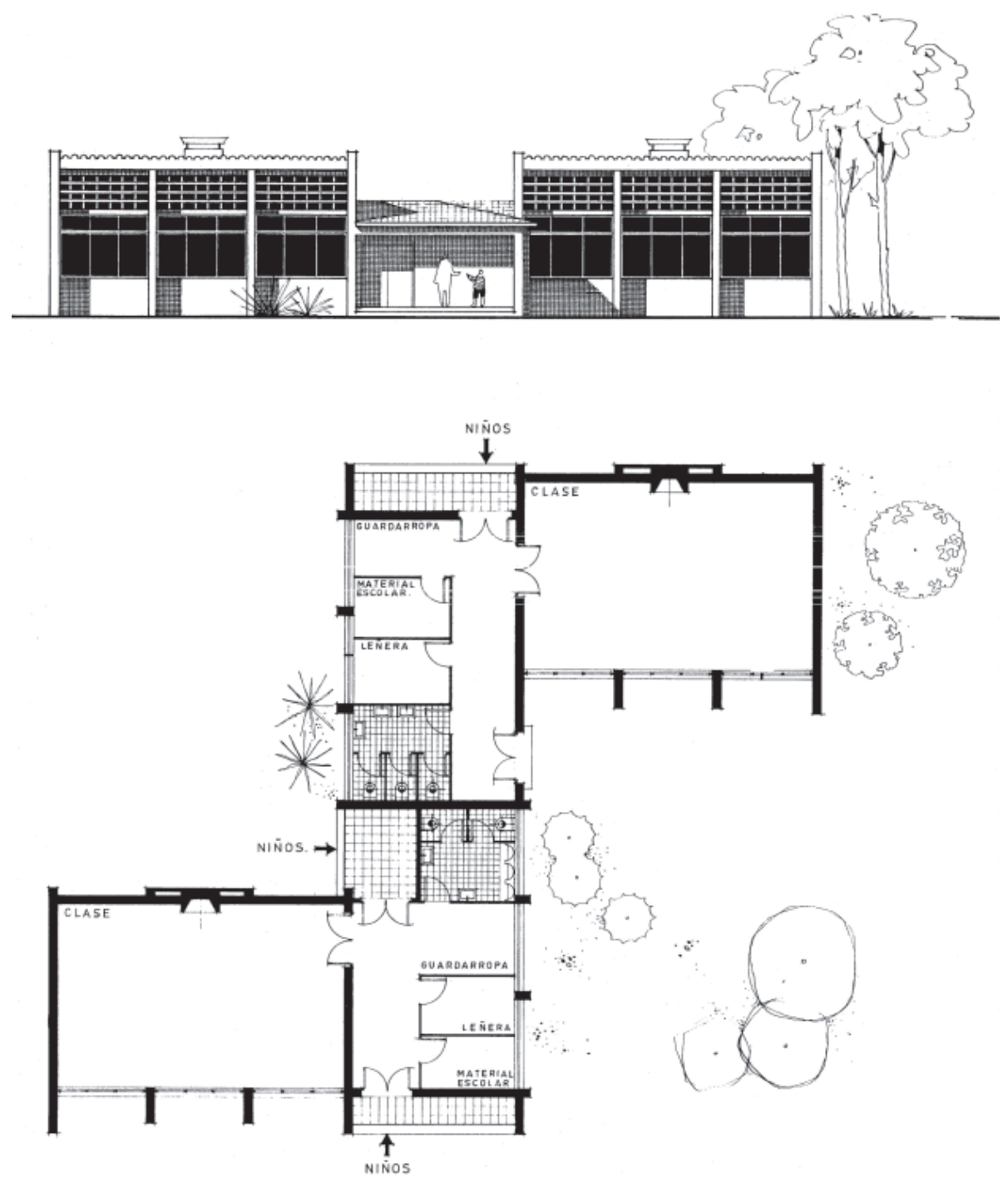

14 Esquema modular de escuelas usado por José Luis Fernández del Amo Moreno en El Trobal (Sevilla), 1962; y por Jesús Ayuso Tejerizo en Nuevos Francos (Salamanca), 1963 


\section{Notas}

1. Antonio Álvaro Tordesillas, «Referencias internacionales en los Pueblos de Colonización espańoles», Ciudades 13 (2010): 183.

2. Los cinco grados en la escala del core que se analizaron y clasificaron en el CIAM fueron: el poblado rural, la aldea con mercado rural o el barrio residencial urbano, la localidad importante rural o el distrito ciudadano urbano, la ciudad y la metrópoli.

3. Sigfied Giedion, A Decade of Contemporary Architecture (Zurich: Girsberger, 1954), 46. Traducción del autor.

4. Citado por Lluis Domènech Girbau, Arquitectura de siempre. Los años 40 en España (Barcelona: Tusquets, 1978).

5. Manuel Calzada Pérez, «Influencias Norteamericanas en el urbanismo del Instituto Nacional de Colonización» en José Manuel Pozo Municio y Javier Martínez González (eds.), La arquitectura norteamericana, motor y espejo de la arquitectura española en el arranque de la modernidad (actas del congreso) (Pamplona: T4 ediciones, 2006), 89.

6. Cf. Alfredo Villanueva Paredes y Jesús Leal Maldonado, La planificación del regadio y los poblados de colonización (Madrid: MAP/MOPU/MAPA, 1991).

7. Cf. Gonzalo de Cárdenas y Rodríguez, «La Reconstrucción Nacional vista desde la Dirección General de Regiones Devastadas», Reconstrucción 1 (1940): 4-7.

8. Cf. Arieh Sharon, Kibbutz+Bauhaus, an architect's way in a New land (Stuttgart: Kramer Verlag, 1976).

9. José Luis Fernández del Amo Moreno, Memoria del Proyecto del pueblo de La Vereda, en la zona del Bembézar (Sevilla) (Madrid: INC, 1963).

10. Miguel Centellas Soler, «Proyectar lo trascendente. Las iglesias de colonización de Fernández del Amo», en Varios autores, Pueblos de colonización durante el franquismo. La arquitectura en la modernización del territorio rural (actas del congreso) (Sevilla: Junta de Andalucía, 2005): 344-356.

\section{Procedencia de las ilustraciones}

Fig. 01-02 y 04-11. Planos y fotografías aéreas del Archivo del INC, San Fernando de Henares (Madrid). Dibujos realizados por el autor. Fig. 03. Alfredo Villanueva Paredes y Jesús Leal Maldonado, La planificación del regadio y los poblados de colonización (Madrid: MAP/MOPU/MAPA, 1991).

Fig. 12. Fotografías realizadas por el autor.

Fig. 13. Villanueva Paredes y Leal Maldonado, La planificación del regadío, y archivo del autor.

Fig. 14. Archivo del INC, San Fernando de Henares (exp. 12631).

\section{Sobre los autores}

Antonio Álvaro Tordesillas. Arquitecto por la ETSA de la Universidad de Valladolid (1999). Doctor Arquitecto (2008), Profesor Ayudante Doctor del Departamento de Urbanismo y Representación de la Arquitectura en la ETSA de Valladolid. Su investigación principal se divide en dos líneas: el análisis y levantamiento arquitectónico y urbano de los Pueblos de Colonización en Espańa, donde tiene un I+D financiado por la JCYL, varios libros y capítulos en libros sobre el tema, así como artículos en revistas indexadas y actas de congresos. La segunda tiene que ver con la asignatura que imparte (Geometría descriptiva) y su posible aportación para la docencia: técnicas de restitución sencillas y de bajo coste. Además de las publicaciones docentes que ha realizado, tiene artículos y actas de congresos en Italia que tratan el tema. Actualmente desarrolla un I+D titulado "Low-cost photogrammetric new tecnic by digital photography; applied to the lifting of works by Chillida and Oteiza".

tordesillas@arq.uva.es

Alberto Meiss. Doctor arquitecto y Profesor de Construcciones Arquitectónicas (Acondicionamiento e Instalaciones) en la ETSA de Valladolid e integrante del Grupo de Investigación "Arquitectura \& Energía". Ponente en cursos y conferencias en universidades, colegios profesionales y congresos sobre el tema de las instalaciones. Ha escrito articulos en revistas científicas y participado en varios proyectos de investigación nacionales. Su línea de investigación se centra en el tema de la ventilación, la eficiencia de los sistemas de acondicionamiento y la repercusión energética en los edificios (Tesis doctoral: "Estudio de la eficiencia de la ventilación a partir de parámetros de diseńo arquitectónico). Profesor e Investigador del Dep. Construcciones Arquitectónicas (sección Instalaciones) y Responsable del Laboratorio de Ventilación HS3 en la E.T.S. Arquitectura de Valladolid.

meiss@arq.uva.es 